TRANSACTIONS OF THE

AMERICAN MATHEMATICAL SOCIETY

Volume 363, Number 6, June 2011, Pages 3057-3069

S 0002-9947(2011)05064-6

Article electronically published on January 26, 2011

\title{
INFINITESIMAL EINSTEIN DEFORMATIONS OF NEARLY KÄHLER METRICS
}

\author{
ANDREI MOROIANU AND UWE SEMMELMANN
}

\begin{abstract}
It is well known that every 6-dimensional strictly nearly Kähler manifold $(M, g, J)$ is Einstein with positive scalar curvature scal $>0$. Moreover, one can show that the space $E$ of co-closed primitive $(1,1)$-forms on $M$ is stable under the Laplace operator $\Delta$. Let $E(\lambda)$ denote the $\lambda$-eigenspace of the restriction of $\Delta$ to $E$. If $M$ is compact, and has normalized scalar curvature scal $=30$, we prove that the moduli space of infinitesimal Einstein deformations of the nearly Kähler metric $g$ is naturally isomorphic to the direct sum $E(2) \oplus E(6) \oplus E(12)$. From Moroianu, Nagy, and Semmelmann (2008), the last summand is itself isomorphic with the moduli space of infinitesimal nearly Kähler deformations.
\end{abstract}

\section{INTRODUCTION}

Nearly Kähler manifolds, introduced by Alfred Gray in the 70s in the framework of weak holonomy, are defined as almost Hermitian manifolds $(M, g, J)$ which are not far from being Kähler in the sense that the covariant derivative of $J$ with respect to the Levi-Civita connection of $g$ is totally skew-symmetric.

The class of nearly Kähler manifolds is clearly stable under Riemannian products. Using the generalization by Richard Cleyton and Andrew Swann of the Berger-Simons holonomy theorem to the case of connections with torsion [3], PaulAndi Nagy showed in 7 that every nearly Kähler manifold is locally a Riemannian product of Kähler manifolds, 3-symmetric spaces, twistor spaces over positive quaternion-Kähler manifolds and 6-dimensional nearly Kähler manifolds. This result shows, in particular, that genuine nearly Kähler geometry only occurs in dimension 6. It turns out that in this dimension, strict (i.e. non-Kähler) nearly Kähler manifolds have several other remarkable features: They carry a real Killing spinor - so they are in particular Einstein manifolds with positive scalar curvature - and they have an $\mathrm{SU}_{3}$ structure whose intrinsic torsion is parallel with respect to the minimal connection (cf. [3]). A strict nearly Kähler structure on a compact 6 -dimensional manifold with normalized scalar curvature scal $=30$ is called a Gray structure.

In [5] we have studied the moduli space $\mathcal{G}$ of infinitesimal deformations of Gray structures on compact 6-dimensional manifolds, and showed that this space is isomorphic to the space $E(12)$, where $E(\lambda)$ denotes the intersection of the $\lambda$-eigenspace of the Laplace operator and the space of co-closed primitive $(1,1)$-forms.

Received by the editors June 10, 2008 and, in revised form, March 30, 2009.

2010 Mathematics Subject Classification. Primary 58H15, 58E30, 53C10, 53C15.

Key words and phrases. Einstein deformations, nearly Kähler manifolds, Gray structures.

(C)2011 American Mathematical Society

Reverts to public domain 28 years from publication 3057 
In the present paper we consider the related problem of describing the moduli space $\mathcal{E}$ of Einstein deformations of a Gray structure. Since every Gray structure is in particular Einstein, one has a priori $\mathcal{E} \supset \mathcal{G}$. Our main result (Theorem 5.1) gives a canonical isomorphism between $\mathcal{E}$ and the direct sum $E(2) \oplus E(6) \oplus E(12)$.

The main idea is the following. The space of infinitesimal Einstein deformation on every compact manifold consists of trace-free symmetric bilinear tensors in a certain eigenspace of a second order elliptic operator called the Lichnerowicz Laplacian $\Delta_{L}$. On a 6-dimensional nearly Kähler manifold, one can decompose every infinitesimal Einstein deformation $H$ (viewed as symmetric endomorphism) into its parts $h$ and $S$ commuting resp. anti-commuting with $J$. Under the $\mathrm{SU}_{3}$ representation, the space of symmetric endomorphisms commuting with $J$ is isomorphic to the space of $(1,1)$-forms and that of symmetric endomorphisms anti-commuting with $J$ is isomorphic to the space of primitive $(2,1)+(1,2)$-forms and one may interpret the eigenvalue equation for $\Delta_{L}$ in terms of the forms $\varphi$ and $\sigma$ corresponding to $h$ and $S$. The problem is that $\Delta_{L}$ does not commute with the isomorphisms above, because $J$ is not parallel with respect to the Levi-Civita connection. It is thus natural to introduce a modified Lichnerowicz operator $\bar{\Delta}_{L}$, corresponding to the canonical Hermitian connection, better adapted to the nearly Kähler setting. It turns out that the eigenvalue equation for $\Delta_{L}$ translates, via $\bar{\Delta}_{L}$, into a differential system for $\varphi$ and $\sigma$ involving the usual form Laplacian, which eventually yields the claimed result.

\section{Preliminaries}

2.1. Notation. In this section we introduce our objects of study and derive several lemmas which will be needed later. Here and henceforth, $\left(M^{2 m}, g, J\right)$ will denote an almost Hermitian manifold with tangent bundle $T M$, cotangent bundle $T^{*} M$ and tensor bundle $\mathcal{T} M$. We denote as usual by $\lambda^{(p, q)+(q, p)} M$ the projection of the complex bundle $\lambda^{(p, q)} M$ onto the real bundle $\lambda^{p+q} M$. The bundle of $g$-symmetric endomorphisms $\operatorname{Sym} M$ splits in a direct $\operatorname{sum} \operatorname{Sym} M=\operatorname{Sym}^{+} M \oplus \operatorname{Sym}^{-} M$, of symmetric endomorphisms commuting resp. anti-commuting with $J$. The trace of every element in $\mathrm{Sym}^{-} M$ is automatically 0 , and $\mathrm{Sym}^{+} M$ decomposes further $\mathrm{Sym}^{+} M=\operatorname{Sym}_{0}^{+} M \oplus\langle\mathrm{id}\rangle$ into its trace-free part and multiples of the identity.

2.2. Nearly Kähler manifolds. An almost Hermitian manifold $\left(M^{2 m}, g, J\right)$ is called nearly Kähler if

$$
\left(\nabla_{X} J\right)(X)=0, \quad \forall X \in T M,
$$

where $\nabla$ denotes the Levi-Civita connection of $g$. The canonical Hermitian connection $\bar{\nabla}$, defined by

$$
\bar{\nabla}_{X} Y:=\nabla_{X} Y-\frac{1}{2} J\left(\nabla_{X} J\right) Y, \quad \forall X \in T M, Y \in \chi(M),
$$

is a $\mathrm{U}_{m}$ connection on $M$ (i.e. $\bar{\nabla} g=0$ and $\bar{\nabla} J=0$ ) with torsion $\bar{T}_{X} Y=$ $-J\left(\nabla_{X} J\right) Y$. A fundamental observation, which - although not explicitly stated goes back to Gray, is the fact that $\bar{\nabla} \bar{T}=0$ on every nearly Kähler manifold (see [1]).

We denote as usual the Kähler form of $M$ by $\omega:=g(J .,$.$) . The tensor \psi^{+}:=$ $\nabla \omega$ is totally skew-symmetric by (2.1). Moreover, since $J^{2}=-\mathrm{id}$, it is easy to check that $\psi^{+}(X, J Y, J Z)=-\psi^{+}(X, Y, Z)$. In other words, $\psi^{+}$is a form of type $(3,0)+(0,3)$. Let us now assume that the dimension of $M$ is $2 m=6$ and that the 
nearly Kähler structure is strict, i.e. $(M, g, J)$ is not Kähler. The form $\psi^{+}$can be seen as the real part of a $\bar{\nabla}$-parallel complex volume form on $M$, so $M$ carries an $\mathrm{SU}_{3}$ structure whose minimal connection (cf. [3]) is exactly $\bar{\nabla}$.

Let $A \in \lambda^{1} M \otimes \operatorname{End} M$ denote the tensor $A_{X}:=J\left(\nabla_{X} J\right)=-\psi_{J X}^{+}$. We will sometimes identify the endomorphism $A_{X}$ with the corresponding form in $\lambda^{(2.0)+(0,2)} M$, e.g. in formula (2.8) below. By definition, we have $\nabla_{X}=\bar{\nabla}_{X}+\frac{1}{2} A_{X}$ on $T M$. In fact this relation can be extended on the whole tensor bundle, provided we use the right extension for $A_{X}$.

2.3. The induced action. On a manifold $M$, every endomorphism $A$ of $T M$ extends as derivation to the tensor bundle $\mathcal{T} M$. In fact if we identify $\operatorname{End}\left(T_{x} M\right)$ with $\mathfrak{g l}(n, \mathbb{R})$ this is precisely the Lie algebra action on the defining representation of $\mathcal{T} M$. We denote by $A_{\star}$ this induced action. For example, we have

$$
A_{\star} \tau=-\tau \circ A, \quad A_{\star} f=A \circ f-f \circ A, \quad \text { for } \tau \in T^{*} M, f \in \operatorname{End} M .
$$

If $(M, g, J)$ is almost Hermitian and $f \in \operatorname{Sym}^{+} M$, let $\tilde{f}$ denote the associated $(1,1)$ form $\tilde{f}:=g(J f .,$.$) , so in particular \tilde{\text { id }}=\omega$, where $\omega$ denotes the Kähler form of $M$. We compute, for later use:

$$
\left(f_{\star} \omega\right)(X, Y)=-\omega(f X, Y)-\omega(X, f Y)=-g(J f X, Y)-g(J X, f Y)=-2 \tilde{f}(X, Y) .
$$

A similar calculation shows that

$$
S_{\star} \omega=0, \quad \forall S \in \mathrm{Sym}^{-} M .
$$

Notice that the map $\operatorname{End} M \rightarrow \operatorname{End}(\mathcal{T} M), A \mapsto A_{\star}$ is a Lie algebra morphism, i.e.

$$
[A, B]_{\star}=\left[A_{\star}, B_{\star}\right], \quad \forall A, B \in \operatorname{End} M,
$$

which can be expressed as

$$
A_{\star}\left(B_{\star} T\right)=\left(A_{\star} B\right)_{\star} T+B_{\star}\left(A_{\star} T\right), \quad \forall A, B \in \operatorname{End} M, T \in \mathcal{T} M .
$$

A convenient way of writing the induced action of $A \in \operatorname{End} M$ on a $p$-form $u$ is

$$
\left.A_{\star} u=-A^{*}\left(e_{i}\right) \wedge e_{i}\right\lrcorner u,
$$

where $A^{*}$ is the adjoint of $A$ and $\left\{e_{i}\right\}$ is a local orthonormal basis of $T M$. Here, as well as in the remaining part of this paper, we adopt the Einstein convention of summation on the repeated subscripts.

Notice that by (2.2), the extensions of $\nabla$ and $\bar{\nabla}$ to the tensor bundle $\mathcal{T} M$ are related by

$$
\bar{\nabla}_{X}=\nabla_{X}-\frac{1}{2}\left(A_{X}\right)_{\star}
$$

2.4. Algebraic results on nearly Kähler manifolds. Assume that $\left(M^{6}, g, J\right)$ is a strict nearly Kähler manifold and that the metric on $M$ is normalized such that scal $=30$. From [4, Theorem 5.2] it follows that for every unit vector $X$, the endomorphism $\nabla_{X} J$ (which vanishes on the 2-plane spanned by $X$ and $J X$ ) defines a complex structure on the orthogonal complement of that 2-plane. Then the same holds for $A_{X}$ (because $A_{X}=-\nabla_{J X} J$ ).

The exterior bundle $\lambda^{2} M$ decomposes into irreducible $\mathrm{SU}_{3}$ components as follows:

$$
\lambda^{2} M \simeq \lambda^{(2,0)+(0,2)} M \oplus \lambda_{0}^{(1,1)} M \oplus \mathbb{R} \omega .
$$


The map $X \mapsto X\lrcorner \psi^{+}$identifies the first summand with $T M$, and $h \mapsto g(J h .,$. defines an isomorphism between $\operatorname{Sym}_{0}^{+} M$ and the second summand.

Similarly, one can decompose $\lambda^{3} M$ into irreducible $\mathrm{SU}_{3}$ components

$$
\lambda^{3} M \simeq \lambda^{(3,0)+(0,3)} M \oplus \lambda_{0}^{(2,1)+(1,2)} M \oplus \lambda^{1} M \wedge \omega .
$$

The first summand is a rank 2 trivial bundle spanned by $\psi^{+}$and its Hodge dual $* \psi^{+}$, and the isomorphism $S \mapsto S_{\star} \psi^{+}$identifies $\mathrm{Sym}^{-} M$ with the second summand.

If $\left\{e_{i}\right\}$ denotes a local orthonormal basis of $T M$, it is straightforward to check the following formulas:

$$
\begin{gathered}
A_{e_{i}} A_{e_{i}}(X)=-4 X, \quad \forall X \in T M . \\
A_{e_{i}} \wedge A_{e_{i}}=2 \omega^{2} .
\end{gathered}
$$

Lemma 2.1. (i) For every $X \in T M$, with corresponding 1-form $X^{b}$ one has

$$
A_{X \star} \psi^{+}=-2 X^{\mathrm{b}} \wedge \omega \text {. }
$$

(ii) If $S$ is a section of $\mathrm{Sym}^{-} M$, then the following formula holds for every $X \in$ $T M$ :

$$
A_{X \star}\left(S_{\star} \psi^{+}\right)=2(S X)^{b} \wedge \omega .
$$

Proof. (i) An easy computation shows:

$$
\begin{aligned}
A_{X \star} \psi^{+} & \left.\left.=A_{X} e_{i} \wedge e_{i}\right\lrcorner \psi^{+}=A_{X} J e_{i} \wedge J e_{i}\right\lrcorner \psi^{+}=A_{J e_{i}} X \wedge A_{e_{i}} \\
& \left.=A_{e_{i}}(J X) \wedge A_{e_{i}}=\frac{1}{2} J X\right\lrcorner\left(A_{e_{i}} \wedge A_{e_{i}}\right) \stackrel{2.8}{=}-2 X \wedge \omega .
\end{aligned}
$$

(ii) The symmetric endomorphism $A_{X \star} S=A_{X} \circ S-S \circ A_{X}$ commutes with $J$ and is trace-free. Consequently, by Schur's Lemma (cf. [5] for a more detailed argument)

$$
\left(A_{X \star} S\right)_{\star} \psi^{+}=0
$$

Notice that if $X^{b}$ is the 1 -form corresponding to $X$ (which we usually identify with $X)$, then $f_{\star} X^{b}=-(f X)^{b}$ for every symmetric endomorphism $f$. We then compute:

$$
\begin{aligned}
A_{X \star}\left(S_{\star} \psi^{+}\right) \stackrel{\sqrt[2.5]{=}}{=}\left(A_{X \star} S\right)_{\star} \psi^{+}+S_{\star}\left(A_{X \star} \psi^{+}\right) \stackrel{2.9}{=}, 2 S_{\star}\left(X^{\mathrm{b}} \wedge \omega\right) \\
=2(S X)^{\mathrm{b}} \wedge \omega-2 X \wedge\left(S_{\star} \omega\right) \stackrel{2.11}{=} 2(S X)^{\mathrm{b}} \wedge \omega .
\end{aligned}
$$

\section{The CURVATURE OPERATOR}

Let $\left(M^{n}, g\right)$ be a Riemannian manifold. The curvature operator $\mathcal{R}: \Lambda^{2} M \rightarrow$ $\Lambda^{2} M$ is defined by the equation $g(\mathcal{R}(X \wedge Y), U \wedge V)=g\left(R_{X, Y} V, U\right)$, for any vector fields $X, Y, U, V$ on $M$, identified with the corresponding 1-forms via the metric. In a local orthonormal frame $\left\{e_{i}\right\}$ it can be written as

$$
\mathcal{R}\left(e_{i} \wedge e_{j}\right)=\frac{1}{2} R_{i j k l} e_{l} \wedge e_{k}=-\frac{1}{2} e_{k} \wedge R_{e_{i}, e_{j}} e_{k} .
$$

Using the identification of 2-vectors and (skew-symmetric) endomorphisms given by $(X \wedge Y)(Z):=g(X, Z) Y-g(Y, Z) X$, formula (3.1) yields $\mathcal{R}(X \wedge Y)(Z)=-R_{X, Y} Z$. Notice that a manifold with curvature operator $\mathcal{R}=c$ id has Ricci curvature $c(n-1)$ and in particular the curvature operator of the sphere is a positive multiple of the identity. 
Let $E M$ be the vector bundle associated to the bundle of orthonormal frames via some representation $\pi: S O(n) \rightarrow \operatorname{Aut}(E)$. Every orthogonal automorphism $f$ of $T M$ defines in a canonical way an automorphism of $E M$, denoted, by a slight abuse of notation, $\pi(f)$. The differential of $\pi$ maps skew-symmetric endomorphisms of $T M$ (or equivalently elements of $\Lambda^{2} M$ ) to endomorphisms of $E M$. The LeviCivita connection of $M$ induces a connection on $E M$ whose curvature $R^{E}$ satisfies $R^{E}(X, Y)=\pi_{*}(R(X, Y))=-\pi_{*}(\mathcal{R}(X \wedge Y))$. Notice that $\pi_{*}(A)$ is exactly $A_{\star}$ in the notation of Section 2 .

We now define the curvature endomorphism $q(R) \in \operatorname{End}(E M)$ as

$$
q(R):=-\frac{1}{2}\left(e_{i} \wedge e_{j}\right)_{\star} \mathcal{R}\left(e_{i} \wedge e_{j}\right)_{\star} .
$$

For example the curvature endomorphism $q(R)$ on the form bundle $E M=\Lambda^{p} M$ satisfies

$$
\left.\left.q(R)=\left(e_{j} \wedge e_{i}\right\lrcorner\right) \circ\left(R_{e_{i}, e_{j}} e_{k} \wedge e_{k}\right\lrcorner\right) .
$$

In particular we have $q(R)=$ Ric on 1-forms, and $q(R)=-$ Ric $_{\star}-2 \mathcal{R}$ on 2 -forms.

It is easy to check that the action of $q(R)$ is compatible with the identification of $\Lambda^{2} M$ with the space of skew-symmetric endomorphisms (and, more generally, with all $\mathrm{SO}_{n}$ equivariant isomorphisms):

Lemma 3.1. Let $\varphi \in \Lambda^{2} M$ be a 2-form with associated skew-symmetric endomorphism $A$, i.e. $\varphi(Y, Z)=g(A Y, Z)$ for any vector fields $Y, Z$. Then

$$
(q(R) \varphi)(Y, Z)=g((q(R) A) Y, Z)
$$

We now return to the case of a 6-dimensional strict nearly Kähler manifold $\left(M^{6}, g, J\right)$ with scalar curvature scal $=30$.

Let $R$ be the curvature of the Levi-Civita connection and let $\bar{R}$ be the curvature of the canonical Hermitian connection $\bar{\nabla}$. The following relation between $R$ and $\bar{R}$ is implicitly contained in [4].

Lemma 3.2. For any tangent vectors $W, X, Y, Z$ one has

$$
\begin{aligned}
R_{W X Y Z}= & \bar{R}_{W X Y Z}-\frac{1}{4}(g(Y, W) g(X, Z)+g(X, Y) g(Z, W) \\
& +3 g(Y, J W) g(J X, Z)-3 g(Y, J X) g(J W, Z)-2 g(X, J W) g(J Y, Z)) .
\end{aligned}
$$

Proof. The stated formula follows using equation (3.1) and the polarization of equation (5.1) from [4]. Note that there is a different sign convention for the curvature tensors in [4].

The Ricci curvature of $\bar{R}$ satisfies $\overline{\mathrm{Ric}}=4 g$. This follows from the formula above and the fact that $\left(M^{6}, g, J\right)$ is Einstein with Ric $=5 g$.

Replacing $R$ by $\bar{R}$ in formula (3.3) yields a curvature endomorphism $q(\bar{R})$. It is easy to check that the curvature operator with respect to $\bar{\nabla}$, denoted by $\overline{\mathcal{R}}$, is a section of $\operatorname{Sym}\left(\Lambda_{0}^{(1,1)} M\right)$ so we can express $q(\bar{R})=-\sum \alpha_{i \star} \overline{\mathcal{R}}\left(\alpha_{i}\right)_{\star}$ for any orthonormal basis $\alpha_{i}$ of $\Lambda_{0}^{(1,1)} M$. Since $\Lambda_{0}^{(1,1)} \mathbb{R}^{6} \simeq \mathfrak{s u}_{3}$, we see that $q(\bar{R})$ preserves all tensor bundles associated to $\mathrm{SU}_{3}$ representations. Moreover, a straightforward computation using the fact that $\alpha_{\star} J=0$ and $\alpha_{\star} \psi^{+}=0$ for every $\alpha \in \mathfrak{s u}_{3}$ yields:

$$
g\left((q(\bar{R}) h) J_{.}, .\right)=q(\bar{R}) \varphi, \quad \text { and } \quad q(\bar{R})\left(S_{\star} \psi^{+}\right)=(q(\bar{R}) S)_{\star} \psi^{+},
$$


for every sections $h \in \operatorname{Sym}^{+} M$ and $S \in \operatorname{Sym}^{-} M$, where $\varphi$ denotes the $(1,1)$-form defined by $\varphi=g(h J .,$.$) .$

The following lemma describes the difference $q(R)-q(\bar{R})$. It is an immediate consequence of the curvature formula in Lemma 3.2. We will denote with Cas $=$ $\frac{1}{2}\left(e_{i} \wedge e_{j}\right)_{\star}\left(e_{i} \wedge e_{j}\right)_{\star}$ the Casimir operator of $\mathfrak{s o}(n)$ acting on the representation $E$ and at the same time the corresponding endomorphism of $E M$.

Lemma 3.3. The difference $q(R)-q(\bar{R}) \in \operatorname{End}(E M)$ is given as

$$
q(R)-q(\bar{R})=-\frac{1}{4} \operatorname{Cas}+\frac{3}{8}\left(e_{i} \wedge e_{j}\right)_{\star}\left(J e_{i} \wedge J e_{j}\right)_{\star}-\frac{1}{8}\left(e_{i} \wedge J e_{i}\right)_{\star}\left(e_{k} \wedge J e_{k}\right)_{\star} .
$$

In the remaining part of this section we will apply Lemma 3.3 in order to compute $q(R)-q(\bar{R})$ on certain spaces of endomorphisms and forms.

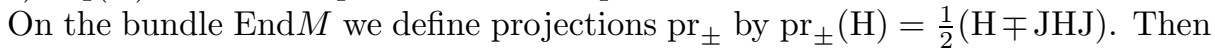
$H=\operatorname{pr}_{+}(\mathrm{H})+\mathrm{pr}_{-}(\mathrm{H})$ is the decomposition of the endomorphism $H$ in a part commuting resp. anti-commuting with $J$.

\section{Proposition 3.4.}

$$
q(R)-q(\bar{R})=\left\{\begin{array}{lll}
3 \text { id } & \text { on } & \mathrm{Sym}^{+} M \\
2 \text { id } & \text { on } & \operatorname{Sym}^{-} M \\
- \text { id } & \text { on } & \Lambda_{0}^{(1,1)} M \\
- \text { id } & \text { on } & \Lambda^{(2,1)+(1,2)} M .
\end{array}\right.
$$

Proof. We start by computing:

$$
\left(e_{i} \wedge e_{j}\right)_{\star}\left(J e_{i} \wedge J e_{j}\right)_{\star}=\left\{\begin{array}{lll}
-2 \mathrm{id} & \text { on } & T M \\
-8 \mathrm{pr}_{-} & \text {on } & \operatorname{Sym} M \\
-8 \mathrm{pr}_{+} & \text {on } & \Lambda_{0}^{2} M .
\end{array}\right.
$$

Indeed for any tangent vector $v \in T M$ we have

$$
\begin{aligned}
\left(e_{i} \wedge e_{j}\right)_{\star}\left(J e_{i} \wedge J e_{j}\right)_{\star} v & =\left(e_{i} \wedge e_{j}\right)_{\star}\left(g\left(J e_{i}, v\right) J e_{j}-g\left(J e_{j}, v\right) J e_{i}\right) \\
& =-2\left(J v \wedge e_{j}\right)_{\star} J e_{j}=-2 v .
\end{aligned}
$$

We now recall that for every skew-symmetric endomorphism $A \in \mathfrak{s o}(T M) \cong \Lambda^{2} M$ and for every $H \in \operatorname{End} M$ we have $A_{\star} H=[A, H]$. Hence, we obtain

$$
\begin{aligned}
\left(e_{i} \wedge e_{j}\right)_{\star}\left(J e_{i} \wedge J e_{j}\right)_{\star} H= & {\left[\left(e_{i} \wedge e_{j}\right),\left[\left(J e_{i} \wedge J e_{j}\right), H\right]\right] } \\
= & \left(e_{i} \wedge e_{j}\right)\left(J e_{i} \wedge J e_{j}\right) H+H\left(e_{i} \wedge e_{j}\right)\left(J e_{i} \wedge J e_{j}\right) \\
& -2\left(e_{i} \wedge e_{j}\right) H\left(J e_{i} \wedge J e_{j}\right) \\
= & -4 H-2\left(e_{i} \wedge e_{j}\right) H\left(J e_{i} \wedge J e_{j}\right) .
\end{aligned}
$$

It remains to compute the endomorphism $B:=\left(e_{i} \wedge e_{j}\right) H\left(J e_{i} \wedge J e_{j}\right)$. Applying it to a vector $Y$ and taking the scalar product with a vector $Z$ we find

$$
\begin{aligned}
g(B Y, Z) & =-g\left(H\left(J e_{i} \wedge J e_{j}\right) Y,\left(e_{i} \wedge e_{j}\right) Z\right) \\
& =-g\left(H\left[g\left(J e_{i}, Y\right) J e_{j}-g\left(J e_{j}, Y\right) J e_{i}\right],\left[g\left(e_{i}, Z\right) e_{j}-g\left(e_{j}, Z\right) e_{i}\right]\right) \\
& =-2\left(g(J Z, Y) g\left(H J e_{j}, e_{j}\right)+g(H J Z, J Y)\right) .
\end{aligned}
$$

Hence the sum $B=\left(e_{i} \wedge e_{j}\right) H\left(J e_{i} \wedge J e_{j}\right)$ equals $2 J H J$ if the endomorphism $H$ is symmetric and $-2 J H J$ if $H$ is skew-symmetric and $\operatorname{tr}(H J)=0$. 
We now compute the second sum of Lemma 3.3 on tangent vectors and endomorphisms. Since $2 J=\left(e_{i} \wedge J e_{i}\right)$ we immediately obtain

$$
\left(e_{i} \wedge J e_{i}\right)_{\star}\left(e_{j} \wedge J e_{j}\right)_{\star}=\left\{\begin{array}{lll}
-4 \mathrm{id} & \text { on } & T M \\
-16 \mathrm{pr}_{-} & \text {on } & \operatorname{End} M .
\end{array}\right.
$$

We next determine the two sums of Lemma 3.3 on the space of 3-forms. Recall the type decomposition

$$
\Lambda^{3} M=\Lambda^{(3,0)+(0,3)} M \oplus \Lambda^{(2,1)+(1,2)} M,
$$

which coincides with the eigenspace decomposition of $\left(J_{\star}\right)^{2}$, with eigenvalue -9 on the first and eigenvalue -1 on the second summand. For any 3 -form $\alpha$ we define a new 3 -form $\hat{\alpha}$ by the formula $\hat{\alpha}(X, Y, Z)=\alpha(J X, J Y, Z)+\alpha(J X, Y, J Z)+$ $\alpha(X, J Y, J Z)$. Then $\left(J_{\star}\right)^{2} \alpha=-3 \alpha+2 \hat{\alpha}$ and the two components of $\Lambda^{3} M$ may also be characterized by

$$
\alpha \in \Lambda^{(3,0)+(0,3)} M \quad \text { if and only if } \quad \hat{\alpha}=-3 \alpha,
$$

and similarly

$$
\alpha \in \Lambda^{(2,1)+(1,2)} M \quad \text { if and only if } \quad \hat{\alpha}=\alpha .
$$

Let $\mathrm{pr}_{3,0}$ and $\mathrm{pr}_{2,1}$ denote the projections onto the two summands of $\Lambda^{3} M$. Then for $\alpha \in \Lambda^{3} M$

$$
\operatorname{pr}_{3,0}(\alpha)=\frac{1}{4}(\alpha-\hat{\alpha}) \quad \text { and } \quad \operatorname{pr}_{2,1}(\alpha)=\frac{1}{4}(3 \alpha+\hat{\alpha}) .
$$

For any 3 -form $\alpha$ we have $\left(e_{i} \wedge J e_{i}\right)_{\star}\left(e_{k} \wedge J e_{k}\right)_{\star} \alpha=4\left(J_{\star}\right)^{2} \alpha=-12 \alpha+8 \hat{\alpha}$, which gives the second sum of Lemma 3.3 and a simple calculation yields $\left(e_{i} \wedge e_{j}\right)_{\star}\left(J e_{i} \wedge J e_{j}\right)_{\star} \alpha=$ $-6 \alpha-4 \hat{\alpha}$ for the first sum.

Recall that in the normalization with $\left\{e_{i} \wedge e_{j}\right\}$ as orthonormal basis of $\Lambda^{2} M \cong$ $\mathfrak{s o}(T M)$, the Casimir operator acts as $-p(n-p)$ id on $\Lambda^{p} M$, and as $-2 n$ id on $\operatorname{Sym} M$. Taking $n=6$ and using the explicit expressions above together with Lemma 3.3 completes the proof.

\section{Comparing Rough Laplacians}

Let $(M, g, J)$ be a strict nearly Kähler manifold with Levi-Civita connection $\nabla$ and canonical Hermitian connection $\bar{\nabla}$. In this section we compare the actions of the rough Laplacians $\nabla^{*} \nabla$ and $\bar{\nabla}^{*} \bar{\nabla}$ on several tensor bundles.

We will perform all calculations below at some fixed point $x \in M$ using a local orthonormal frame $\left\{e_{i}\right\}$ which is $\nabla$-parallel at $x$. On any tensor bundle on $M$ we can write $\nabla^{*} \nabla=-\nabla_{e_{i}} \nabla_{e_{i}}$ and because $\bar{\nabla}_{e_{i}} e_{i}=\nabla_{e_{i}} e_{i}-\frac{1}{2} J\left(\nabla_{e_{i}} J\right) e_{i}=0$, we also have $\bar{\nabla}^{*} \bar{\nabla}=-\bar{\nabla}_{e_{i}} \bar{\nabla}_{e_{i}}$. We are interested in the operator $P:=\nabla^{*} \nabla-\bar{\nabla}^{*} \bar{\nabla}$. Using (2.6) and the fact that the tensor $A:=J \nabla J$ is $\bar{\nabla}$-parallel, we have

$$
\begin{aligned}
P & =-\nabla_{e_{i}} \nabla_{e_{i}}+\bar{\nabla}_{e_{i}} \bar{\nabla}_{e_{i}}=-\left(\bar{\nabla}_{e_{i}}+\frac{1}{2} A_{e_{i} \star}\right)\left(\bar{\nabla}_{e_{i}}+\frac{1}{2} A_{e_{i} \star}\right)+\bar{\nabla}_{e_{i}} \bar{\nabla}_{e_{i}} \\
& =-\frac{1}{4} A_{e_{i} \star} A_{e_{i} \star}-A_{e_{i} \star} \bar{\nabla}_{e_{i}} .
\end{aligned}
$$

We now compute the action of the two operators occurring in the previous formula on several tensor bundles which are of interest in the deformation problem. 
Lemma 4.1. Let $\varphi, \sigma, h$ and $S$ be sections of $\Lambda_{0}^{(1,1)} M, \Lambda_{0}^{(2,1)+(1,2)} M$, $\operatorname{Sym}^{+} M$ and $\mathrm{Sym}^{-} M$ respectively. Then

$$
\begin{gathered}
A_{e_{i} \star}\left(A_{e_{i} \star} \varphi\right)=-4 \varphi . \\
A_{e_{i} \star}\left(A_{e_{i} \star} \sigma\right)=-4 \sigma . \\
A_{e_{i} \star}\left(A_{e_{i} \star} h\right)=-12 h . \\
A_{e_{i} \star}\left(A_{e_{i} \star} S\right)=-8 S .
\end{gathered}
$$

Proof. Let $X \in T M$ be a tangent vector. Since $A_{X}$ and $\varphi$ are 2 -forms of type $(2,0)+(0,2)$ and $(1,1)$ respectively, we get

$$
A_{X}\left(e_{k}, e_{j}\right) \varphi\left(e_{k}, e_{j}\right)=2\left\langle A_{X}, \varphi\right\rangle=0, \quad \forall X \in T M .
$$

We then compute

$$
\begin{aligned}
& \left.\left.A_{e_{i} \star}\left(A_{e_{i} \star} \varphi\right)=A_{e_{i}} e_{j} \wedge e_{j}\right\lrcorner\left(A_{e_{i}} e_{k} \wedge e_{k}\right\lrcorner \varphi\right) \\
& \left.=\left\langle A_{e_{i}} e_{k}, e_{j}\right\rangle A_{e_{i}} e_{j} \wedge\left(e_{k}\right\lrcorner \varphi\right)-\varphi\left(e_{k}, e_{j}\right) A_{e_{i}} e_{j} \wedge A_{e_{i}} e_{k} \\
& \left.\left.\stackrel{4.5}{=} A_{e_{i}}^{2}\left(e_{k}\right) \wedge\left(e_{k}\right\lrcorner \varphi\right)-e_{j}\right\lrcorner\left(\varphi\left(e_{k}, e_{j}\right) A_{e_{i}} \wedge A_{e_{i}} e_{k}\right) \\
& \left.\left.\left.\stackrel{2.7}{=}-4 e_{k} \wedge\left(e_{k}\right\lrcorner \varphi\right)-\frac{1}{2} e_{j}\right\lrcorner e_{k}\right\lrcorner\left(A_{e_{i}} \wedge A_{e_{i}} \varphi\left(e_{k}, e_{j}\right)\right) \\
& \left.\left.\left.\stackrel{2.8}{=}-8 \varphi-e_{j}\right\lrcorner e_{k}\right\lrcorner\left(\omega^{2} \varphi\left(e_{k}, e_{j}\right)\right)=-8 \varphi-2 e_{j}\right\lrcorner\left(J e_{k} \wedge \omega \varphi\left(e_{k}, e_{j}\right)\right) \\
& \left.=-8 \varphi-2 \omega \varphi\left(e_{k}, J e_{k}\right)+2 J e_{k} \wedge J e_{j} \varphi\left(e_{k}, e_{j}\right)\right) \\
& \left.=-8 \varphi+2 e_{k} \wedge e_{j} \varphi\left(e_{k}, e_{j}\right)\right)=-8 \varphi+4 \varphi=-4 \varphi \text {. }
\end{aligned}
$$

In order to prove (4.2), we express $\sigma$ as $\sigma=S_{\star} \psi^{+}$for some section $S$ of $\mathrm{Sym}^{-} M$. Using (2.10) we obtain

$$
\begin{aligned}
A_{e_{i} \star}\left(A_{e_{i} \star} \sigma\right) & =2 A_{e_{i} \star}\left(S e_{i} \wedge \omega\right)=2 S e_{i} \wedge\left(A_{e_{i} \star} \omega\right)=2 S e_{i} \wedge J \psi_{e_{i}}^{+} e_{j} \wedge J e_{j} \\
& =-2 S e_{i} \wedge \psi_{e_{i}}^{+} J e_{j} \wedge J e_{j}=4 S e_{i} \wedge \psi_{e_{i}}^{+}=-4 S_{\star} \psi^{+}=-4 \sigma .
\end{aligned}
$$

Now, for every endomorphism $H$ of $T M$, we have

$$
A_{e_{i} \star}\left(A_{e_{i} \star} H\right)=A_{e_{i}}^{2} H+H A_{e_{i}}^{2}-2 A_{e_{i}} H A_{e_{i}} \stackrel{2.77}{=}-8 H-2 A_{e_{i}} H A_{e_{i}} .
$$

If $h \in \operatorname{Sym}^{+} M$, let $\varphi(.,)=.g(J h .,$.$) be its associated (1,1)$-form. By (4.1) we have for every tangent vectors $X, Y$

$$
\begin{aligned}
& -4 \varphi(X, Y)=A_{e_{i} \star}\left(A_{e_{i} \star} \varphi\right)(X, Y) \\
& =\varphi\left(A_{e_{i}}^{2} X, Y\right)+\varphi\left(X, A_{e_{i}}^{2} Y\right)+2 \varphi\left(A_{e_{i}} X, A_{e_{i}} Y\right) \\
& \stackrel{2.77}{=}-8 \varphi(X, Y)+2 g\left(h J A_{e_{i}} X, A_{e_{i}} Y\right) \\
& =-8 \varphi(X, Y)+2 g\left(J A_{e_{i}} h A_{e_{i}} X, Y\right),
\end{aligned}
$$

whence $A_{e_{i}} h A_{e_{i}}=2 h$. This, together with (4.6), yields (4.3). If $S \in \mathrm{Sym}^{-} M$, using the fact that $A_{J X}=A_{X} \circ J=-J \circ A_{X}$ for every $X$, we can write

$$
A_{e_{i}} S A_{e_{i}}=A_{J e_{i}} S A_{J e_{i}}=-A_{e_{i}} J S J A_{e_{i}}=-A_{e_{i}} S A_{e_{i}},
$$

which together with (4.6) yields (4.4). 
Lemma 4.2. The following relations hold:

$$
\begin{gathered}
\left.e_{i}\right\lrcorner\left(A_{e_{i} \star} \varphi\right)=0, \quad \forall \varphi \in \Lambda_{0}^{(1,1)} M . \\
\left(A_{e_{i} \star} h\right)\left(e_{i}\right)=0, \quad \forall h \in \operatorname{Sym}_{0}^{+} M . \\
e_{i} \wedge\left(A_{e_{i} \star} \varphi\right)=0, \quad \forall \varphi \in \Lambda_{0}^{(1,1)} M . \\
\left.e_{i}\right\lrcorner\left(A_{i \star}\left(S_{\star} \psi^{+}\right)\right)=0, \quad \forall S \in \operatorname{Sym}^{-} M .
\end{gathered}
$$

Proof. Simple application of the Schur Lemma, taking into account the decomposition of the exterior bundles into irreducible components with respect to the $\mathrm{SU}_{3}$ action.

Lemma 4.3. Let $\varphi$ and $S$ be sections of $\Lambda_{0}^{(1,1)} M$ and $\mathrm{Sym}^{-} M$ respectively. If $h$ and $\sigma$ are defined as usual by $g(J h .,):.=\varphi(.,$.$) and \sigma:=S_{\star} \psi^{+}$, then

$$
\begin{gathered}
\left.A_{e_{i} \star} \bar{\nabla}_{e_{i}} \varphi=-(J \delta \varphi)\right\lrcorner \psi^{+} . \\
A_{e_{i} \star} \bar{\nabla}_{e_{i}} \sigma=-2 \delta S \wedge \omega . \\
\left(A_{e_{i} \star} \bar{\nabla}_{e_{i}} h\right)_{\star} \psi^{+}=-2 \delta h \wedge \omega-4 d \varphi . \\
\left.A_{e_{i} \star} \bar{\nabla}_{e_{i}} S=(\delta S\lrcorner \psi^{+}+\delta\left(S_{\star} \psi^{+}\right)\right) \circ J .
\end{gathered}
$$

Here $\delta$ denotes the co-differential on exterior forms and the divergence operator whenever applied to symmetric endomorphisms.

Proof. Since $A_{e_{i}}$ anti-commutes with $J$ and $\bar{\nabla}_{e_{i}} \varphi$ is of type $(1,1)$, it follows that $A_{e_{i} \star} \bar{\nabla}_{e_{i}} \varphi$ is a form of type $(2,0)+(0,2)$, so there exists a vector field $\alpha$ such that $\left.A_{e_{i} \star} \bar{\nabla}_{e_{i}} \varphi=\alpha\right\lrcorner \psi^{+}$. In order to find $\alpha$, we use the relation $\left.(\alpha\lrcorner \psi^{+}\right) \wedge \psi^{+}=\alpha \wedge \omega^{2}$ (see [5]) and compute:

$$
\begin{aligned}
\alpha \wedge \omega^{2} & \left.=(\alpha\lrcorner \psi^{+}\right) \wedge \psi^{+}=\left(A_{e_{i} \star} \bar{\nabla}_{e_{i}} \varphi\right) \wedge \psi^{+} \\
& =A_{e_{i} \star}\left(\left(\bar{\nabla}_{e_{i}} \varphi\right) \wedge \psi^{+}\right)-\bar{\nabla}_{e_{i}} \varphi \wedge\left(A_{e_{i} \star} \psi^{+}\right) \stackrel{(2.9)}{=} 2 \bar{\nabla}_{e_{i}} \varphi \wedge e_{i} \wedge \omega \\
& \left.\left.\left.=-\bar{\nabla}_{e_{i}} \varphi \wedge\left(J e_{i}\right\lrcorner \omega^{2}\right)=-J e_{i}\right\lrcorner\left(\bar{\nabla}_{e_{i}} \varphi \wedge \omega^{2}\right)+\left(J e_{i}\right\lrcorner \bar{\nabla}_{e_{i}} \varphi\right) \wedge \omega^{2} \\
& \left.\left.=J\left(e_{i}\right\lrcorner \bar{\nabla}_{e_{i}} \varphi\right) \wedge \omega^{2} \stackrel{4.77}{=} J\left(e_{i}\right\lrcorner \nabla_{e_{i}} \varphi\right) \wedge \omega^{2}=-J \delta \varphi \wedge \omega^{2},
\end{aligned}
$$

so $\alpha=-J \delta \varphi$, thus proving (4.11). Using the fact that $\psi^{+}$is $\bar{\nabla}$-parallel, we get:

$$
\begin{array}{ccc}
A_{e_{i} \star} \bar{\nabla}_{e_{i}} \sigma & = & A_{e_{i} \star}\left(\bar{\nabla}_{e_{i}} S_{\star} \psi^{+}\right) \stackrel{2.5}{=}\left(A_{e_{i} \star} \bar{\nabla}_{e_{i}} S\right)_{\star} \psi^{+}+\bar{\nabla}_{e_{i}} S_{\star}\left(A_{e_{i} \star} \psi^{+}\right) \\
& \underline{2.9}, \underline{\underline{2.11}}-2 \bar{\nabla}_{e_{i}} S_{\star}\left(e_{i} \wedge \omega\right) \stackrel{(2.4)}{=} 2\left(\bar{\nabla}_{e_{i}} S\right) e_{i} \wedge \omega=-2 \delta S \wedge \omega .
\end{array}
$$

This proves (4.12). We next use (4.8) to write

$$
\delta h=-\left(\nabla_{e_{i}} h\right) e_{i}=-\left(\bar{\nabla}_{e_{i}} h\right) e_{i}
$$

whence

$$
\begin{aligned}
& \left(A_{e_{i} \star} \bar{\nabla}_{e_{i}} h\right)_{\star} \psi^{+} \stackrel{2.5}{=} A_{e_{i} \star}\left(\left(\bar{\nabla}_{e_{i}} h\right)_{\star} \psi^{+}\right)-\left(\bar{\nabla}_{e_{i}} h\right)_{\star}\left(A_{e_{i} \star} \psi^{+}\right) \\
& \stackrel{\sqrt[2.9]{=}}{=} 2\left(\bar{\nabla}_{e_{i}} h\right)_{\star}\left(e_{i} \wedge \omega\right)=2\left(\bar{\nabla}_{e_{i}} h\right) e_{i} \wedge \omega+2 e_{i} \wedge\left(\left(\bar{\nabla}_{e_{i}} h\right)_{\star} \omega\right) \\
& \stackrel{2.30}{=}-2 \delta h \wedge \omega-4 e_{i} \wedge \bar{\nabla}_{e_{i}} \varphi=-2 \delta h \wedge \omega-4 d \varphi \text {. }
\end{aligned}
$$


In order to check (4.14) we first compute

$$
\begin{aligned}
\delta\left(S_{\star} \psi^{+}\right) & \left.\left.\left.=-e_{i}\right\lrcorner \nabla_{e_{i}}\left(S_{\star} \psi^{+}\right) \stackrel{4.10}{=}-e_{i}\right\lrcorner \bar{\nabla}_{e_{i}}\left(S_{\star} \psi^{+}\right)=e_{i}\right\lrcorner \bar{\nabla}_{e_{i}}\left(S\left(e_{j}\right) \wedge \psi_{e_{j}}^{+}\right) \\
& \left.\left.=e_{i}\right\lrcorner\left(\left(\bar{\nabla}_{e_{i}} S\right) e_{j} \wedge \psi_{e_{j}}^{+}\right)=-g\left(\delta S, e_{j}\right) \psi_{e_{j}}^{+}-\left(\bar{\nabla}_{e_{i}} S\right) e_{j} \wedge\left(e_{i}\right\lrcorner \psi_{e_{j}}^{+}\right) \\
& \left.=-\delta S\lrcorner \psi^{+}+\left(e_{i}\right\lrcorner \psi_{e_{j}}^{+}\right) \wedge\left(\bar{\nabla}_{e_{i}} S\right) e_{j} .
\end{aligned}
$$

Let $B$ denote the endomorphism of $T M$ corresponding to the 2-form $\delta\left(S_{\star} \psi^{+}\right)+$ $\delta S\lrcorner \psi^{+}$. By the calculation above we get

$$
\begin{aligned}
B(X) & \left.=\left(\left(e_{i}\right\lrcorner \psi_{e_{j}}^{+}\right) \wedge\left(\bar{\nabla}_{e_{i}} S\right) e_{j}\right)(X) \\
& \left.=\psi^{+}\left(e_{j}, e_{i}, X\right)\left(\bar{\nabla}_{e_{i}} S\right) e_{j}-\left(\bar{\nabla}_{e_{i}} S\right)\left(e_{j}, X\right)\left(e_{i}\right\lrcorner \psi_{e_{j}}^{+}\right) \\
& =\left(\bar{\nabla}_{e_{i}} S\right)\left(\psi_{e_{i}}^{+} X\right)+\psi_{e_{i}}^{+}\left(\left(\bar{\nabla}_{e_{i}} S\right) X\right)=\left(\bar{\nabla}_{e_{i}} S\right)\left(A_{e_{i}} J X\right)+A_{e_{i}}\left(J\left(\bar{\nabla}_{e_{i}} S\right) X\right) \\
& =\left(\bar{\nabla}_{e_{i}} S\right)\left(A_{e_{i}} J X\right)-A_{e_{i}}\left(\left(\bar{\nabla}_{e_{i}} S\right) J X\right)=-\left(A_{e_{i} \star}\left(\bar{\nabla}_{e_{i}} S\right)\right)(J X) .
\end{aligned}
$$

Replacing $X$ by $J X$ yields (4.14).

From Lemma 4.1 and Lemma 4.3 we infer directly

Corollary 4.4. Let $\varphi$ and $S$ be sections of $\Lambda_{0}^{(1,1)} M$ and $\mathrm{Sym}^{-} M$ respectively. If $h$ and $\sigma$ are defined by $g(J h .,):.=\varphi(.,$.$) and \sigma:=S_{\star} \psi^{+}$, then

$$
\begin{gathered}
\left.\left(\nabla^{*} \nabla-\bar{\nabla}^{*} \bar{\nabla}\right) \varphi=\varphi+(J \delta \varphi)\right\lrcorner \psi^{+} . \\
\left(\nabla^{*} \nabla-\bar{\nabla}^{*} \bar{\nabla}\right) \sigma=\sigma+2 \delta S \wedge \omega . \\
\left(\nabla^{*} \nabla-\bar{\nabla}^{*} \bar{\nabla}\right) h=3 h+s, \\
\text { where } s \in \operatorname{Sym}^{-} M, \text { and } s_{\star} \psi^{+}=2 \delta h \wedge \omega+4 d \varphi . \\
\left.\left(\nabla^{*} \nabla-\bar{\nabla}^{*} \bar{\nabla}\right) S=2 S-(\delta S\lrcorner \psi^{+}+\delta \sigma\right) \circ J .
\end{gathered}
$$

Finally, we obtain the invariance of the space of primitive co-closed $(1,1)$-forms under the Laplace operator:

Proposition 4.5. If $\varphi$ is a co-closed section of $\Lambda_{0}^{(1,1)} M$, then the same holds for $\Delta \varphi$.

Proof. The 2-form $\Delta \varphi$ is clearly co-closed since $\varphi$ is co-closed. Using (4.16), Proposition 3.4 and the classical Weitzenböck formula on 2-forms yield

$$
\Delta \varphi=\left(\nabla^{*} \nabla+q(R)\right) \varphi=\left(\bar{\nabla}^{*} \bar{\nabla}+q(\bar{R})\right) \varphi .
$$

The last term is a section of $\Lambda_{0}^{(1,1)} M$ since both $\bar{\nabla}$ and $q(\bar{R})$ preserve this space.

\section{The Moduli SPACE of Einstein Deformations}

We now have all the ingredients for the main result of this paper:

Theorem 5.1. Let $\left(M^{6}, g, J\right)$ be a Gray manifold. Then the moduli space of infinitesimal Einstein deformations of $g$ is isomorphic to the direct sum of the spaces of primitive co-closed $(1,1)$-eigenforms of the Laplace operator for the eigenvalues 2 , 6 and 12.

Proof. Let $g$ be an Einstein metric with Ric $=E g$. From [2, Theorem 12.30], the space of infinitesimal Einstein deformations of $g$ is isomorphic to the set of symmetric trace-free endomorphisms $H$ of $T M$ such that $\delta H=0$ and such that 
$\Delta_{L} H=2 E H$, where $\Delta_{L}=\nabla^{*} \nabla+q(R)$ is the so-called Lichnerowicz Laplacian $\Delta_{L}$. Remark that $q(R)=2 \stackrel{\circ}{R}+2 E$ id in the notation of [2].

In our present situation the Einstein constant equals $E=5$, so the space of infinitesimal Einstein deformations of $g$ is isomorphic to the set of $H \in \operatorname{Sym} M$ with $\delta H=0=\operatorname{tr} H$ such that

$$
\left(\nabla^{*} \nabla+q(R)\right) H=10 H
$$

Let $h:=\operatorname{pr}_{+} \mathrm{H}$ and $S:=\mathrm{pr}_{-} \mathrm{H}$ denote the projections of $H$ onto $\operatorname{Sym}^{ \pm} M$. We define the primitive $(1,1)$-form $\varphi(.,):.=g(J h .,$.$) and the 3$-form $\sigma:=S_{\star} \psi^{+}$. The key idea is to express (5.1) in terms of an exterior differential system for $\varphi$ and $\sigma$. Using Proposition 3.4 and Corollary 4.4, (5.1) becomes

$\left.\left(\bar{\nabla}^{*} \bar{\nabla}+q(\bar{R})\right)(h+S)=10(h+S)-(3 h+s)-\left(2 S-(\delta S\lrcorner \psi^{+}+\delta \sigma\right) \circ J\right)-3 h-2 S$,

where $s$ is the section of $\mathrm{Sym}^{-} M$ defined in the second part of (4.18). Since the operator $\left(\bar{\nabla}^{*} \bar{\nabla}+q(\bar{R})\right)$ preserves the decomposition $\operatorname{Sym} M=\operatorname{Sym}^{+} M \oplus \operatorname{Sym}^{-} M$, the previous equation is equivalent to the system

$$
\left\{\begin{array}{l}
\left(\bar{\nabla}^{*} \bar{\nabla}+q(\bar{R})\right) h=4 h+\delta \sigma \circ J \\
\left(\bar{\nabla}^{*} \bar{\nabla}+q(\bar{R})\right) S=6 S-s \\
\delta S=0 .
\end{array}\right.
$$

Taking the composition with $J$ and using (3.4), the first equation of (5.2) becomes

$$
\left(\bar{\nabla}^{*} \bar{\nabla}+q(\bar{R})\right) \varphi=4 \varphi-\delta \sigma .
$$

Similarly, taking the action on $\psi^{+}$and using (3.4) and the definition of $s$, the second equation of (5.2) becomes

$$
\left(\bar{\nabla}^{*} \bar{\nabla}+q(\bar{R})\right) \sigma=6 \sigma-2 \delta h \wedge \omega-4 d \varphi .
$$

Notice that $\delta h=\delta H-\delta S=0$, which can also be seen by examining the algebraic types in equation (5.4). From (4.15) we get

$$
0=\delta h=-\left(\bar{\nabla}_{e_{i}} h\right) e_{i}=\left(\bar{\nabla}_{e_{i}} J \varphi\right) e_{i}=J\left(\bar{\nabla}_{e_{i}} \varphi\right) e_{i}=-J \delta \varphi,
$$

so finally the system (5.2) is equivalent to

$$
\left\{\begin{array}{l}
\left(\bar{\nabla}^{*} \bar{\nabla}+q(\bar{R})\right) \varphi=4 \varphi-\delta \sigma, \\
\left(\bar{\nabla}^{*} \bar{\nabla}+q(\bar{R})\right) \sigma=6 \sigma-4 d \varphi \\
\delta \varphi=0
\end{array}\right.
$$

Using Proposition 3.4 together with the equations (4.16) and (4.17) (keeping in mind that $\delta S=0$ and $\delta \varphi=0)$ we get the two identities $\left(\bar{\nabla}^{*} \bar{\nabla}+q(\bar{R})\right) \varphi=\left(\nabla^{*} \nabla+\right.$ $q(R)) \varphi$ and $\left(\bar{\nabla}^{*} \bar{\nabla}+q(\bar{R})\right) \sigma=\left(\nabla^{*} \nabla+q(R)\right) \sigma$. Hence the classical Weitzenböck formula for the Laplace operator on forms implies that (5.5) is equivalent to

$$
\left\{\begin{array}{l}
\Delta \varphi=4 \varphi-\delta \sigma \\
\Delta \sigma=6 \sigma-4 d \varphi \\
\delta \varphi=0
\end{array}\right.
$$

Lemma 5.2. Let $E(\lambda)$ be the $\lambda$-eigenspace of $\Delta$ restricted to the space of co-closed primitive $(1,1)$-forms. Then the space of solutions of the system (5.6) is isomorphic 
to the direct sum $E(2) \oplus E(6) \oplus E(12)$. The isomorphism can be written explicitly as

$$
(\varphi, \sigma) \stackrel{\Psi}{\mapsto}(8 \varphi+\delta \sigma, * d \sigma, 2 \varphi-\delta \sigma) \in E(2) \oplus E(6) \oplus E(12)
$$

with inverse

$$
(\alpha, \beta, \gamma) \in E(2) \oplus E(6) \oplus E(12) \mapsto\left(\frac{\Phi+\gamma}{10}, \frac{3 d \alpha-5 * d \beta-2 d \gamma}{30}\right) .
$$

Proof. The first thing to check is the fact that $\Phi$ and $\Psi$ take values in the right spaces.

Let $(\alpha, \beta, \gamma) \in E(2) \oplus E(6) \oplus E(12)$ and $(\varphi, \sigma):=\Phi(\alpha, \beta, \gamma)$. From Lemma 4.4 in [5], the exterior derivative of every co-closed primitive $(1,1)$-form is a primitive $(2,1)+(1,2)$-form. Thus $\varphi \in \Omega_{0}^{(1,1)} M$ and $\sigma \in \Omega_{0}^{(2,1)+(1,2)} M$. A simple calculation shows that $(\varphi, \sigma)$ satisfy the system (5.6).

Conversely, let $(\varphi, \sigma)$ be a solution of (5.6) and $(\alpha, \beta, \gamma):=\Psi(\varphi, \sigma)$. Clearly $\alpha, \beta$ and $\gamma$ are co-closed. From Proposition 4.5 and the first equation of (5.6) we see that $\delta \sigma$ is a section of $\Lambda_{0}^{(1,1)} M$, so the same holds for $\alpha$ and $\gamma$. The fact that $* d \sigma$ is a section of the same bundle follows from Lemma 4.3 in 5 . A direct check shows that $\Delta \alpha=2 \alpha, \Delta \beta=6 \beta$ and $\Delta \gamma=12 \gamma$.

Finally, it is straightforward to check that $\Phi$ and $\Psi$ are inverse to each other. This proves the lemma and the theorem.

In order to apply this result, one should try to compute the spectrum of the Laplacian on 2-forms on some explicit compact nearly Kähler 6-dimensional manifolds. Besides the sphere $S^{6}$ - which has no infinitesimal Einstein deformations (cf. [2]) - the only known examples are 3-symmetric spaces $\mathbb{C P}^{3}=\mathrm{SO}_{5} / \mathrm{U}_{2}$, $F(1,2)=\mathrm{SU}_{3} / U_{1} \times \mathrm{U}_{1}$ and $\mathrm{SU}_{2} \times \mathrm{SU}_{2}=\mathrm{SU}_{2} \times \mathrm{SU}_{2} \times \mathrm{SU}_{2} / \Delta$.

Computations of the Laplace spectrum using the Peter-Weyl theorem show that $E(2)$ and $E(6)$ vanish on each of these spaces. Moreover, $E(12)$ vanishes on $\mathbb{C P}^{3}$ and on $\mathrm{SU}_{2} \times \mathrm{SU}_{2}$, and is 8-dimensional on $F(1,2)$ (cf. [6] ). As a consequence of these facts, we deduce:

(1) every infinitesimal Einstein deformation of the 6-dimensional 3-symmetric spaces is an infinitesimal Gray deformation (cf. [5]);

(2) the nearly Kähler structure on $\mathbb{C P}^{3}$ and on $\mathrm{SU}_{2} \times \mathrm{SU}_{2}$ is rigid;

(3) there is an 8-dimensional space of infinitesimal deformations of the nearly Kähler structure on $F(1,2)$.

\section{REFERENCES}

1. F. Belgun, A. Moroianu, Nearly Kähler 6-manifolds with reduced holonomy, Ann. Global Anal. Geom. 19 (2001), 307-319. MR.1842572 (2002f:53083)

2. A. Besse, Einstein manifolds, Ergebnisse der Mathematik und ihrer Grenzgebiete (3) 10. Springer-Verlag, Berlin, (1987). MR867684 (88f:53087)

3. R. Cleyton, A. Swann, Einstein metrics via intrinsic or parallel torsion, Math. Z. 247 (2004), 513-528. MR2114426(2005i:53054)

4. A. Gray, The structure of nearly Kähler manifolds, Math. Ann. 223 (1976), 233-248. MR0417965 (54:6010)

5. A. Moroianu, P.-A. Nagy, U. Semmelmann, Deformations of nearly Kähler structures, Pacific J. Math. 235 (2008), 57-72. MR2379770 (2009b:53038) 
6. A. Moroianu, U. Semmelmann, The Hermitian Laplace operator on nearly Kähler manifolds, Commun. Math. Phys. 294 (2010), 251-272. MR2575483 (2011a:58064)

7. P.-A. NAGY, Nearly Kähler geometry and Riemannian foliations, Asian J. Math. 3 (2002), 481-504. MR1946344 (2003m:53043)

CMls, École Polytechnique, UMr 7640 du CNRS, 91128 Palaiseau, France

E-mail address: am@math.polytechnique.fr

Mathematisches Institut, Universität zu Köln, Weyertal 86-90, D-50931 Köln, GerMANY

E-mail address: uwe.semmelmann@math.uni-koeln.de

Current address: Department of Mathematics, University of Stuttgart, Pfaffenwaldring 57, D-70569 Stuttgart, Germany

E-mail address: uwe.semmelmann@mathematik.uni-stuttgart.de 